\title{
Evidence of Action-at-a-Distance in Experiments with Individual Particles
}

\author{
M. E. Burgos \\ Departamento de Física, Universidad de Los Andes, Mérida, Venezuela \\ Email: mburgos25@gmail.com
}

Received 29 July 2015; accepted 22 September 2015; published 25 September 2015

Copyright (C) 2015 by author and Scientific Research Publishing Inc.

This work is licensed under the Creative Commons Attribution International License (CC BY). http://creativecommons.org/licenses/by/4.0/

c) (i) Open Access

\begin{abstract}
We make a brief historical revision of action-at-a-distance in quantum mechanics. Non-locality has been mostly related to systems of two particles in an entangled state. We show that this effect is also apparent in some experiments with individual particles. An easily performed experiment in this regard is introduced.
\end{abstract}

\section{Keywords}

\section{Foundations of Quantum Mechanics, The Measurement Problem in Quantum Mechanics}

\section{Introduction}

In the Fifth Solvay Congress (1927) A. Einstein analyzed the following experiment. A particle (photon or electron) impinges normally on a diaphragm with slit 0 so that the $\psi$-wave associated with the particle is diffracted in 0 . A scintillation-screen (or photographic film) in the shape of a hemisphere is placed behind 0 so as to show the arrival of a particle (Figure 1), an event whose probability of occurrence is measured by the "intensity" of the diffracted spherical waves at the point under consideration. Einstein declared:

"According to viewpoint $I$ the de Broglie-Schrödinger waves do not represent one individual particle but rather an ensemble of particles distributed in space. Accordingly, the theory provides information not on an individual process but rather on an ensemble of them. Thus $|\psi(r)|^{2}$ expresses the probability (probability density) that there exists at $r$ some particle of the ensemble" [1].

He continued: “According to viewpoint II quantum mechanics is considered as a complete theory of individual processes; each particle moving toward the screen is described as a wave packet which, after diffraction, arrives at a certain point $\mathrm{P}$ on the screen, and $|\psi(r)|^{2}$ expresses the probability (probability density) that at a given moment one and the same particle shows its presence at $r .$. If $|\psi|^{2}$ is interpreted according to II, then, as long as no localization has been effected, the particle must be considered as potentially present with almost constant 


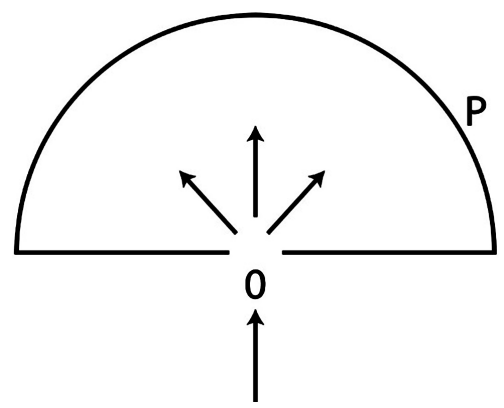

Figure 1. The experiment analyzed by Einstein in the 5th Solvay Congress.

probability over the whole area of the screen; however, as soon as it is localized, a peculiar action-at-a-distance must be assumed to take place which prevents the continuously distributed wave in space from producing an effect at two places on the screen" [1].

"It seems to me," he concluded, "that this difficulty cannot be overcome unless the description of the process in terms of the Schrödinger wave is supplemented by some detailed specification of the localization of the particle during its propagation... If one works only with Schrödinger waves, the interpretation II of $|\psi|^{2}$, I think, contradicts the postulate of relativity" [1].

This experiment displays "the wave-particle duality": even if the solution of the Schrödinger equation describes a wave, no fraction of a lump but an indivisible particle is detected. According to Einstein, the instantaneous projection (collapse, reduction, or quantum jump) leading a non-localized state to a quite-well localized state is a peculiar kind of action-at-a-distance.

Assuming that the process in terms of Schrödinger waves should be supplemented by some detailed specification of the localization of the particle during its propagation, Einstein succeeded to avoid the notion of action-at-a-distance. Concerning this issue L. Ballentine points out: "he argued that a particle should always be thought of as possessing a definite though perhaps unknown position, even when no such definite position is described by the wave function $\psi$ " [2]. This idea, compatible with viewpoint $I$, is at the origin of the Statistical Interpretation of Quantum Mechanics. In this theory, "a pure state (and hence also a general state) provides a description of certain statistical properties of an ensemble of similarly prepared systems, but need not provide a complete description of an individual system” [3].

By contrast, the first postulate of orthodox (ordinary or standard) quantum mechanics establishes that the wave function (or state vector) completely describes the state of the system. This last theory, also called Copenhagen Interpretation, proved stunningly successful [4]. This is the main reason why most physicists and chemists adopt orthodox quantum mechanics, even if it implies a peculiar kind of action-at-a-distance and other strange features that we are not going to refer to in this paper. A critical review of these matters has been recently published [5].

\section{Non-Locality Related to a System of Two Particles in an Entangled State}

In 1935, A. Einstein, B. Podolsky and N. Rosen (EPR) published the well-known article "Can Quantum-Mechanical Description of Physical Reality Be Considered Complete?” [6]. In this paper they deal with a system of two particles in an entangled state. We should stress the contrast with Einstein analysis in the Fifth Solvay Congress, where he refers to one particle. This difference is crucial to the present study, considering that since the publication of the EPR article non-locality has been mostly associated to systems of particles in entangled states. On the contrary, we analyze action-at-a-distance in processes involving a single particle.

In $1964 \mathrm{~J}$. Bell proved a theorem stating that a local hidden variable theory cannot reproduce all statistical predictions of quantum mechanics [7]: He showed that, in the framework of any deterministic and local theory, the correlations between some properties of two particles should satisfy an inequality (Bell's inequality) which could be violated if the two particles were in an entangled state. He concluded that "in a theory in which parameters are added to quantum mechanics to determine the results of individual measurements, without changing the statistical predictions, there must be a mechanism whereby the setting of one measuring device can influence 
the reading of another instrument, however remote. Moreover, the signal involved must propagate instantaneously, so that such a theory could not be Lorentz invariant" [7].

In the following years many experiments aiming to test Bell's inequalities were performed, yielding results which violate them and compatible with quantum mechanical predictions [8]-[13]. The door was then open to explore an even more strange and fascinating phenomenon: quantum teleportation [14]. Because it depends on classical communication, which cannot proceed faster than the speed of light, teleportation cannot be used for superluminal transport or communication. The current record distance for quantum teleportation is $143 \mathrm{~km}$ with photons, and $21 \mathrm{~m}$ with material systems.

These astonishing results have induced many scientists to consider action-at-a-distance as a real effect. But performing experiments with a system of particles in an entangled state is a complicated business, requiring techniques not accessible to everybody. In Section 4 we describe an easily performed experiment in laboratories of optics, facilitating results to a wider public which cannot be explained without invoking non-locality.

Before concluding this section let us come back to the EPR paper. It is frequently called the EPR paradox, although we should refer to the EPR theorem. F. Laloë states it as follow: "if the predictions of quantum mechanics are correct (even for systems made of remote correlated particles) and if physical reality can be described in a local (or separable) way, then quantum mechanics is necessarily incomplete: some elements of reality exists in Nature that are ignored by this theory" [15]. Laloë adds: "The theorem is valid, and has been scrutinized by many scientists who have found no flaw in its derivation...” [15].

Let us stress that 1) orthodox quantum mechanics has proved to be a very successful theory, with apparently no exceptions to its predictions; and 2) the first postulate of this theory states that the $\psi$-wave gives a complete description of an individual particle's state. This couple of facts provides a good reason to suspect that locality is a questionable matter. Our next step will deal with action-at-a-distance in experiments involving individual particles.

\section{Action-at-a-Distance in Some Well-Known Experiments}

The authors we quote here do not mention action-at-a-distance in the experiments they refer to; they focus on other features: wave-particle duality, step of the process where the collapse occurs and loss of information.

In his Lectures on Physics R. Feynman considers a somewhat modified apparatus of the Stern-Gerlach (S-G) type [16]. It consists of a sequence of three high-gradients magnets; see Figure 2(a). The first one (on the left) is just the usual S-G magnet and splits the incoming beam of spin $1 / 2$ particles into two separate beams. The second magnet has the same cross section as the first, but is twice as long and the polarity of its magnetic field is opposite the field in magnet 1 . The second magnet pushes each beam in the opposite direction and bends them back toward the axis. The third magnet is just like the first, and brings the two beams back together again, so that leaves the exit hole along the axis. Possible paths of spin $1 / 2$ particles leaving the oven $O$ are illustrated in Figure 2(b).

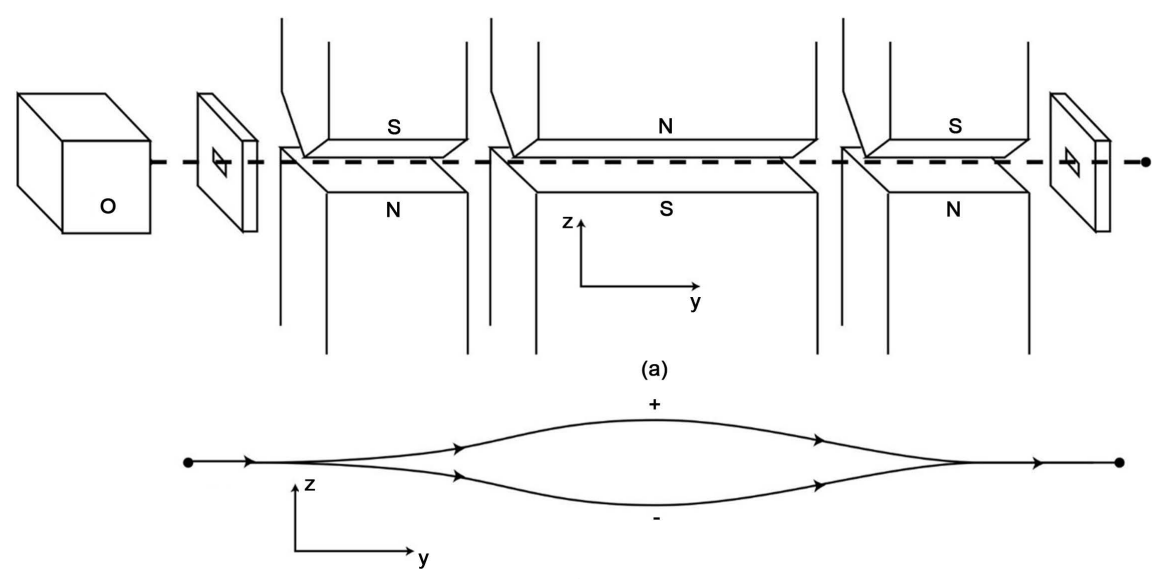

(b)

Figure 2. (a) An imagined modification of a Stern-Gerlach apparatus; (b) The paths of spin $1 / 2$ particles. 
Let $S$ be the operator representing the $z$-component of a spin $1 / 2$ measured in units of $\hbar / 2$. Its eigenvalue equation is $\boldsymbol{S}\left|\varphi_{ \pm}\right\rangle= \pm\left|\varphi_{ \pm}\right\rangle$. A spin 1/2 particle entering this modified S-G in the spin state

$$
|\Psi\rangle=\left\langle\varphi_{+} \mid \Psi\right\rangle\left|\varphi_{+}\right\rangle+\left\langle\varphi_{-} \mid \Psi\right\rangle\left|\varphi_{-}\right\rangle
$$

exits it in the same state, whatever is the state $|\Psi\rangle$, as if the device was not there. If we place a mask in the middle of the apparatus blocking one of the beams, e.g. the lower path, all the particles entering this device in the state $|\Psi\rangle$ exit it in the state $\left|\varphi_{+}\right\rangle$independent of the particular $|\Psi\rangle$ chosen.

Concerning a similar experiment (the difference being that he refers to spin-one particles) Feynman asserts: "Some people would say that... we have 'lost the information' about the previous state $[|\Psi\rangle]$ because we have 'disturbed' the atoms when we separate them into [two] beams in the apparatus... But this is not true. The past information is not lost by the separation into [two] beams, but by the blocking masks that are put in...” [16].

D. Ellerman, a contemporary author, emphasizes: "ordinarily texts represent the S-G apparatus as separating particles into spin eigenstates... But as in our other examples, the apparatus does not project the particles to eigenstates... It is the detectors or blockers that cause the collapse or projections to eigenstates, not the prior separation apparatus" [17].

D. Bes, another contemporary author, analyzes some consequence of quantum principles in the form of thought experiments [18]. Particles leave the oven $O$ in unknown linear combinations $|\Psi\rangle$ of $\left|\varphi_{+}\right\rangle$and $\left|\varphi_{-}\right\rangle$states given by (1). They are collimated and move along the $y$-axis of a sequence of S-G apparatuses like that illustrated in Figure 2. Each separate beam may be masked off at the half way point. Such a device is sketched in Figure 3(a) with the $\left|\varphi_{-}\right\rangle$beam masked off. It will be called a $\varphi$-filter and enclosed within a box drawn with continuous lines.

The particles are prepared in the filtered state $\left|\varphi_{+}\right\rangle$, by preventing particles in the state $\left|\varphi_{-}\right\rangle$from leaving the first filter. In the last stage of the experimental setup another filter is inserted as part of the detector, in order to measure the degree of filtration. The detector includes also a photographic plate which records the arriving particles and is observed by an experimentalist, as shown in Figure 3(b).

Let $\boldsymbol{R}$ be the operator representing the $x$-component of the spin $1 / 2$ particle, measured in units of $\hbar / 2$. Its eigenvalue equation is $\left.\boldsymbol{R}\left|\chi_{ \pm}\right\rangle= \pm \chi_{ \pm}\right\rangle$. These states can be written as linear combinations of $\left|\varphi_{ \pm}\right\rangle$as follows

$$
\begin{aligned}
& \left|\chi_{+}\right\rangle=\left\langle\varphi_{+} \mid \chi_{+}\right\rangle\left|\varphi_{+}\right\rangle+\left\langle\varphi_{-} \mid \chi_{+}\right\rangle\left|\varphi_{-}\right\rangle=(1 / \sqrt{2})\left|\varphi_{+}\right\rangle+(1 / \sqrt{2})\left|\varphi_{-}\right\rangle \\
& \left|\chi_{-}\right\rangle=\left\langle\varphi_{+} \mid \chi_{-}\right\rangle\left|\varphi_{+}\right\rangle+\left\langle\varphi_{-} \mid \chi_{-}\right\rangle\left|\varphi_{-}\right\rangle=(1 / \sqrt{2})\left|\varphi_{+}\right\rangle+(1 / \sqrt{2})\left|\varphi_{-}\right\rangle
\end{aligned}
$$

(a)

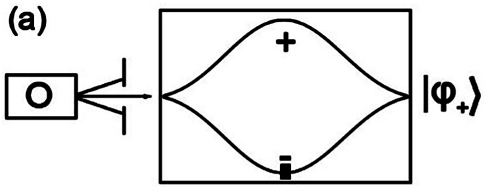

(c)
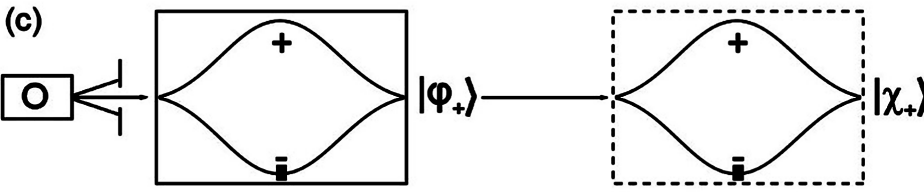

(b)
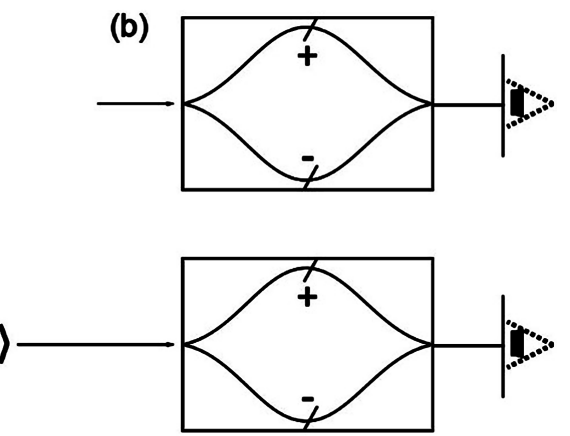

(d)

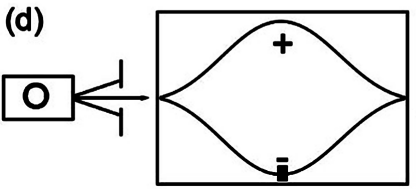

$\left|\varphi_{+}\right\rangle$

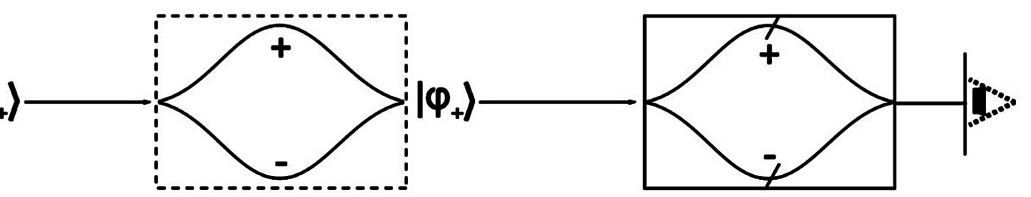

Figure 3. Two quantum mechanical thought experiments: (a) Schematic representation of a $\varphi$-filter; (b) Detector including a filter, photo plate and observer; (c) Experiment A; (d) Experiment B. The vertical bars denote fixed path blockings, while the diagonal bars indicate path that can be opened or closed. For each experiment we perform a measurement in which the upper channel of the detector is open and the down channel blocked, and another measurement with the opposite features. 
Bes then deals with two experiments "which yields results that are spectacular different from classical expectations" [18].

Experiment A (Figure 3(c)): Particles leave the first filter in the state $\left|\varphi_{+}\right\rangle$(with the spin pointing along the positive $z$-axis) and the second filter in the state $\left\langle\chi_{+}\right\rangle$(with the spin pointing along the positive $x$-axis). The modification of the second filter so that it can block the particles in either of the states $\left.\chi_{ \pm}\right\rangle$amounts to a rotation of a $\varphi$-filter by an angle $\pi / 2$ around the $y$-axis. A $\varphi$-filter rotated in this way will be called a $\chi$-filter and represented by a dashed box.

A particle entering the second filter in the state $\left|\varphi_{+}\right\rangle$reorients itself within the second one, a $\chi$-filter. Since the $\left|\chi_{-}\right\rangle$channel of the second filter is blocked (see Figure 3(c)), the particle is either absorbed with probability $\left|\left\langle\varphi_{+} \mid \chi_{-}\right\rangle\right|^{2}=1 / 2$ or is projected into the state $\left|\chi_{+}\right\rangle$(and transmitted) with probability $\left|\left\langle\varphi_{+} \mid \chi_{+}\right\rangle\right|^{2}=1 / 2$. The detector measures the number of particles exiting in one of the $\left|\varphi_{ \pm}\right\rangle$states. Bes stresses that "both components may emerge from the detector filter, in spite of the fact that the fraction of the beam in the $\left|\varphi_{-}\right\rangle$state was annihilated inside the first filter" [18].

Experiment B (Figure 3(d)): The device is the same as in Experiment A except for the absence of the mask in the second filter so that both channels are now open. A particle entering the second filter in the state $\left|\varphi_{+}\right\rangle$exits it in the same state $\left|\varphi_{+}\right\rangle$, as if the device was not there. According to Bes, "the result of this last experiment is equivalent to an interference pattern. Classically, such patterns are associated with waves. However, unlike the case of waves, particles here are always detected as lumps of the same size on a screen placed in front of the exit side of the detector filter. No fractions of a lump are ever detected, as befits the behavior of indivisible particles. Therefore, these experiments display wave-particle duality" [18].

Where do we see action-at-a-distance effects in these experiments? In the first place, let us refer to Experiments A and B. In both devices particles enter the second filter in the state $\left|\varphi_{+}\right\rangle$. Since the path $\left\langle\chi_{-}\right\rangle$in Experiment A is blocked, particles exiting the second filter cannot take a different channel from $\left|\chi_{+}\right\rangle$. Were it possible to discard every kind of action-at-distance in these experiments, the change in channel $\left.\chi_{-}\right\rangle$consisting in the suppression of the blocking mask should yield no effect on something happening in the remote channel $\left\langle\chi_{+}\right\rangle$. As this is not the case, we are forced to conclude that a particle entering the second filter does not take either one or the other channel but, in some way, both of them. But if a particle taking both channels is blocked in one of the channels, it would be entirely blocked. Thus the particle must change suddenly from being partly in one channel and partly in the other to being entirely in one of them. This is a kind of action-at-a-distance.

Needless to say that the same kind of action-at-a-distance is present in the modified S-G analyzed by Feynman and in that referred to by Ellerman. This effect frequently ignored and the attribution of a trajectory to atoms going through a S-G-device is perhaps at the origin of the fallacy pointed out by Ellerman.

\section{Action-at-a-Distance in an Experiment Easy to Perform}

This experiment is similar to another one we suggested some years ago [19]. Its advantage is that it is even easier to perform. All you need is a Michelson-Morley interferometer, a source of monochromatic light, a convergent lens and four polarizations filters.

The polarizations filters are introduced in the device as shown in Figure 4. Due to lens $L$ the beam entering the interferometer is parallel to the optical axis. The two internal filters $\mathrm{F}_{\mathrm{V}}$ and $\mathrm{F}_{\mathrm{H}}$ allow the passage of light with vertical and horizontal polarization, respectively. The filter $\mathrm{F}_{0}$ after $\mathrm{L}$ is oblique at $\alpha$ (the angle between the transmission line of $\mathrm{F}_{0}$ and the horizontal) with $\alpha=\pi / 4$. The filter before the eye $\mathrm{F}_{\mathrm{E}}$ may be rotated, or removed.

The results are:

1) If $F_{E}$ is vertical, horizontal or absent, there is no interference image.

2) If $\mathrm{F}_{\mathrm{E}}$ is oblique at $\beta$ (the angle between the transmission line of $\mathrm{F}_{\mathrm{E}}$ and the horizontal), the interference image, named Newton's rings, emerges. A maximum of visibility is attained for $\beta=\pi / 4$. Increasing $\beta$ even more, Newton's rings fade away and completely disappear when $\beta=\pi / 2$. When $\beta>\pi / 2$ Newton's rings appear again and the visibility reaches its maximum for $\beta=3 \pi / 4$.

More than 200 years ago, Fresnel and Arago stated four laws governing the superposition of light waves from experimental results. According to the fourth law, "two rays of light polarized at right angles and then brought into the same plane of polarization interfere like ordinary light if they were originally polarized in the same plane” [20]. Our results are then in agreement with this law and their interpretation does not present any difficulty as long as the light is considered as made of classical waves. The problem arises if, following P. Dirac, we take as valid "the description which quantum mechanics provides of the interference of photons” [21]. 


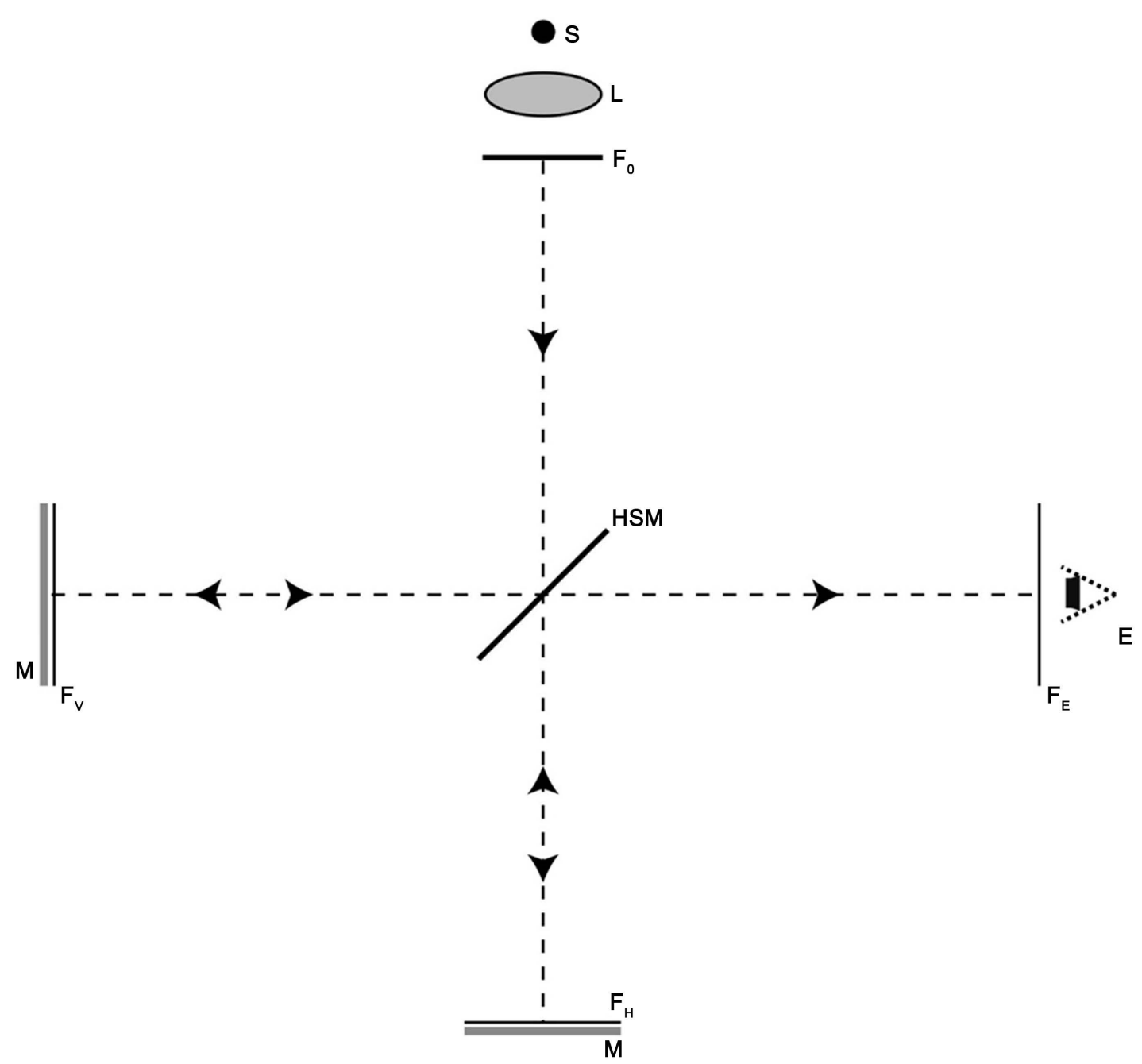

Figure 4. View from the top of a modified Michelson-Morley interferometer. S: source of light; L: convergent lens; $\mathrm{M}$ : mirrors; HSM: half-silvered mirror. $\mathrm{F}_{0}, \mathrm{~F}_{\mathrm{V}}, \mathrm{F}_{\mathrm{H}}$ and $\mathrm{F}_{\mathrm{E}}$ : polarization filters; $\mathrm{E}$ : eye.

In his book The principles of Quantum Mechanics, Dirac says: "Let us take a definite experiment demonstrating interference. Suppose we have a beam of light which is passed through some kind of interferometer, so that it gets split up into two components and the two components are subsequently made to interfere. We may... take an incident beam consisting of only a single photon and inquire what will happen to it as it goes to the apparatus... we must now describe the photon as going partly into each of the two components into which the incident beam is split" [21].

He continues: "Let us consider what happens when we determine the energy in one of the components. The result of such a determination must be either the whole photon or nothing at all. Thus the photon must change suddenly from being partly in one beam and partly in the other to being entirely in one of the beams..." [21]. He adds: "So long as the photon is partly in one beam and partly in the other, interference can occur when the two beams are superposed..." [21]. And finally, at the end of the Section Interference of photons: "The new theory [i.e. quantum mechanics], which connects the wave function with probabilities for one photon, gets over the difficulty [previously mentioned] by making each photon go partly into each of the two components. Each photon then interferes only with itself. Interference between two different photons never occurs" [21].

Following Dirac we say that each photon entering the interferometer goes partly into each of the two components into which the incident beam is split. Now, each photon has three possibilities: being stopped by the filter $\mathrm{F}_{\mathrm{V}}$, being stopped by the filter $\mathrm{F}_{\mathrm{H}}$, or being transmitted. If the photon is not stopped it remains partly in one beam (in a vertical polarization state) and partly in the other (in a horizontal polarization state). So interference 
occurs when the two beams are superposed and brought into the same plane of polarization (thanks to $\mathrm{F}_{\mathrm{E}}$ ). But if the photon is absorbed by one of the filters (either $\mathrm{F}_{\mathrm{V}}$ or $\mathrm{F}_{\mathrm{H}}$ ) it must change suddenly from being partly in one beam and partly in the other to being entirely in the beam stopped by the filter. Action-at-a-distance becomes experimentally evident.

\section{Concluding Remarks}

On one hand, according to EPR theorem, "if the predictions of quantum mechanics are correct and if physical reality can be described in a local way, quantum mechanics is necessarily incomplete” [15]. On the other hand, 1) orthodox quantum mechanics has proved to be a very successful theory, with no exceptions to its predictions; and 2) the first postulate of this theory states that the $\psi$-wave gives a complete description of an individual particle's state. This couple of facts constitutes a good reason to suspect that locality is a questionable matter.

Trying to contribute a solution to this issue, in this paper we have dealt with action-at-a-distance in experiments with individual particles.

Finally, we introduce an experiment of interference with polarization filters. Differing from studies with systems of particles in entangled states, which require techniques not accessible to everybody, the experiment we describe here is very easy to perform in laboratories of optics and facilitates to a wider public results which cannot be explained without invoking non-locality.

\section{Acknowledgements}

I am indebted to Professor J. C. Centeno for fruitful discussions. I thank C. Valero for his help with the figures.

\section{References}

[1] Jammer, M. (1974) The Philosophy of Quantum Mechanics. John Willey \& Sons, New York, 115-116.

[2] Ballentine, L.E. (1972) AJP, 40, 1763-1771.

[3] Ballentine, L.E. (1970) Reviews of Modern Physics, 42, 358-381. http://dx.doi.org/10.1103/RevModPhys.42.358

[4] Tegmar, M. and Wheeler, J. (2001) Scientific American, 284, 68-75. http://dx.doi.org/10.1038/scientificamerican0201-68

[5] Burgos, M.E. (2015) The Measurement Problem in Quantum Mechanics Revisited. In: Pahlavani, M., Ed., Selected Topics in Applications of Quantum Mechanics, INTECH, Croatia, 137-173. http://dx.doi.org/10.5772/59209

[6] Einstein, A., Podolsky, B. and Rosen, N. (1935) Physical Review, 47, 777-780. http://dx.doi.org/10.1103/PhysRev.47.777

[7] Bell, J.S. (1964) Physics, 1, 195-200.

[8] Clauser, J.F., Horne, M.A., Shimony, A. and Holt, R.A. (1969) Physical Review Letters, 23, 880-884. http://dx.doi.org/10.1103/PhysRevLett.23.880

[9] Freedman, S.J. and Clauser, J.F. (1972) Physical Review Letters, 28, 938-941. http://dx.doi.org/10.1103/PhysRevLett.28.938

[10] Clauser, J.F. and Horne, M.A. (1974) Physical Review D, 10, 526-535. http://dx.doi.org/10.1103/PhysRevD.10.526

[11] Aspect, A., Grangier, P. and Roger, G. (1981) Physical Review Letters, 47, 460-463. http://dx.doi.org/10.1103/PhysRevLett.47.460

[12] Aspect, A., Grangierand, P. and Roger, G. (1982) Physical Review Letters, 49, 91-94. http://dx.doi.org/10.1103/PhysRevLett.49.91

[13] Tittel, W., Brendel, J., Zbinden, H. and Gisin, N. (1998) Physical Review Letters, 81, 3563-3566. http://dx.doi.org/10.1103/PhysRevLett.81.3563

[14] Wikipedia, The Free Encyclopedia: “Quantum Teleportation.”

[15] Laloë, F. (2001) American Journal of Physics, 69, 655-701. http://dx.doi.org/10.1119/1.1356698

[16] Feynman, R.P., Leighton, R.B. and Sands, M. (1963) Lectures on Physics. Volume III, Addison-Wesley, Reading.

[17] Ellerman, D. (2011) A Very Common Fallacy in Quantum Mechanics: Superposition, Delayed Choice, Quantum Erasers, Retrocausality, and All That. http://arxiv.org/pdf/1112.4522.pdf

[18] Bes, D.R. (2004) Quantum Mechanics. Springer, Berlin, 12-15. http://dx.doi.org/10.1007/978-3-662-05384-3 
[19] Peres, A. (1993) Quantum Theory: Concepts and Methods. Kluwer Academic Publishers, Dordrecht, 62.

[20] Kanseri, B., Bisht, N.S., Kandpal, H.C. and Rath, S. (2008) American Journal of Physics, 76, 39. http://dx.doi.org/10.1119/1.2794349

[21] Dirac, P.A.M. (1958) The Principles of Quantum Mechanics. Clarendon Press, Oxford, 7-9. 